\title{
POLEN FÓSIL DE ISLA REY JORGE (ANTÁRTICA) Y CHILE CONTINENTAL, AFIN A PROTEACEAE CHILENAS.
}

\author{
FOSSIL POLLEN ON KING GEORGE ISLAND (ANTARCTICA) AND CONTINENTAL \\ CHILE WITH AFFINITY TO CURRENT CHILEAN PROTEACEAE.
}

Ingrid Hebel ${ }^{1} \&$ Teresa Torres ${ }^{2}$

\begin{abstract}
RESUMEN
Se realizó un estudio morfológico de palinomorfos fósiles provenientes de rocas sedimentarias terciarias de la península Fildes, isla Rey Jorge, archipiélago de las Shetland del Sur, Antártica y de dos localidades terciarias de Chile continental. El objetivo es la identificación y descripción de los granos de polen fósil, así como la comparación con las Proteaceae chilenas. De las formas fósiles afines a Proteaceae, ocho pertenecen a Proteacidites, tres a Propylipollis y dos a Triorites. Los palinomorfos afines a especies actuales provienen, principalmente, de sedimentos del Mioceno de Chile continental y del Eoceno Superior de la isla Rey Jorge, Antártica. Se establecen las afinidades de Propylipollis concretus y Proteacidites pseudomoides con Gevuina avellana; Proteacidites aff callosus y Propylipollis sp.1 con Lomatia hirsuta; Proteacidites aff callosus, Proteacidites cf. subscabratus y Proteacidites harrisii con Orites myrtoidea; y Propylipollis microverrucatus con Lomatia ferruginea. El resto no serían afines a ninguna especie actual de Proteaceae.
\end{abstract}

Palabras clave: Palinología, Taxonomía, Descripciones, Análisis Comparativo, Terciario, Paleobotánica.

\section{ABSTRACT}

The morphology of fossil pollen from sedimentary, tertiary rocks on Fildes Peninsula, King George Island, South Shetland Islands, Antarctica and from two locations on continental Chile was studied. The grains were identified, described and compared with current Chilean Proteaceae. Eight of the fossils forms belonged to the genus Proteacidites three to Propylipollis and two to Triorites. Those pollen forms with affinity to current species come mainly in Miocene sediments from continental Chile and from the Upper

1 Escuela de Ciencias Agrícolas y Acuícolas, Facultad de Ciencias, Universidad de Magallanes, Punta Arenas, Chile. ingrid. hebel@umag.cl

2 Laboratorio de Paleobotánica, Facultad de Ciencias Agrarias y Forestales, Universidad de Chile. Casilla 1004, Santiago, Chile. 
Eocene from King George Island. Affinity was established for Propylipollis concretus and Proteacidites pseudomoides with Geruina avellane; Proteacidites aff callosus and Propylipollis sp. 1 with Lomatia hirsuta; Proteacidites aff callosus, Proteacidites cf. subscabratus and Proteacidites harrisii with Orites myrtoidea; and Propylipollis microverrucatus with Lomatia ferruginea. None of the rest are current species of Proteaceae.

Key words: Palynology, Taxonomy, Descriptions, Comparative Analysis, Tertiary Palaeobotany.

\section{INTRODUCCIÓN}

Durante el Cretácico Inferior, 116 millones de años antes del presente (116 Ma AP), evolucionaron las Angiospermae o Magnoliophyta, y aquellos linajes que se establecieron, en el cierre del Cretácico, en el sur de Gondwana. Según Hooker (1847), citado por Dettmann (1992), los datos paleobotánicos han sido cruciales para entender el desarrollo de la flora actual de las regiones australes, y han verificado que ésta incluye fragmentos de una flora que antiguamente ocupaba el gran continente del sur. Muller (1981) citado por Hill \& Scriven (1995), señala que Proteaceae es una de las más importantes, junto a Araucariaceae, Fagaceae, Podocarpaceae, presentes en Chile. De ella, se obtuvo uno de los registros de granos de polen más antiguos en el norte de Gondwana, ya a partir del Cretácico (Cenomaniano-Turoniano, $90 \mathrm{Ma} \mathrm{AP}$ ). La historia de polen fósil sugiere que los ancestros en Gondwana tienen una historia de dispersión antes del Paleoceno (Martin 1995). Australia y Nueva Zelandia fueron los centros de irradiación. Ésta fue mayor durante el Paleoceno-Eoceno, al encontrarse la mayoría de los registros en sedimentos de ese período. Entre las diversas asociaciones halladas en los registros de polen fósil de Proteaceae de la fuente Otway (sudeste de Australia), hay polen afin a las siguientes subfamilias: Persoonioideae, con el género Persoonia; Proteoideae, con los géneros Adenanthos, Beauprea, Stirlingia; Carnarvonioideae, con el género Carnarvonia; Grevilleoideae, con los géneros Grevillea, Telopea, Macadamia, Gevuina/ Hicksbachia y Knightia.

En otros trabajos sobre la flora del Terciario en Australia se comunica la presencia de representantes de otros géneros, además de los ya anteriormente señalados, como Banksia, Banksieaephyllum, Beaupreaidites, Conospermites, Dryandra, Dryandroides, Hakea, Lomatia, Proteacidites, Proteoides, Rhopala, Rhopalophyllum (Duigan 1950).
Carpenter (1994), provee una sinopsis de registros de granos de polen fósil terciarios de Proteaceae, que incluye especímenes afines a Orites, Darlingia y Stenocarpus u Oreocallis del Oligoceno-Mioceno, los que son similares a especies actuales que crecen en bosques tropical-húmedos en el norte de Queensland, Australia. En Nueva Zelandia, el autor notó que las macrofloras con cutículas dispersas del Mioceno, contenían taxa similares a Gevuina/Hicksbeachia y Macadamia, mientras Pole (1992), registra microfósiles de Orites y Banksia en sedimentos del Eoceno.

Desde el Pleistoceno Inferior hasta la mitad del mismo, se encuentran otros géneros pertenecientes a la familia Proteaceae de afinidades subalpinas en las partes bajas del oeste de Tasmania, reconocidos casi exclusivamente por registros de polen fósil, tales como Banksia, Grevillea, Agastachys, cf Lomatia, Orites, cf Persoonia, Telopea (Jordan et al. 1995).

En relación con la península Antártica e islas adyacentes hay registros de granos de polen fósil de la familia en estudio, en el cerro Fósil, península Fildes, isla Rey Jorge (Torres \& Méon 1990, Li 1992), bahía Almirantazgo, isla Rey Jorge (Stuchlik 1981), isla Seymour (Askin 1989, Askin 1990, Torres 19901), península Antártica (Dettman 1989), regiones del mar de Weddell (Mohr 1990). En lo que respecta a la edad, Askin (1990) da a conocer información sobre polen fósil del Campaniano-Maastrichtiano-Daniano recolectado en la isla Seymour, Antártica, que corresponden a: Proteacidites, Propylipollis, Cranwellipollis, Beaupreaidites, pertenecientes a Proteaceae. Además informa sobre Peninsulapollis, presumiblemente perteneciente a Proteaceae.

1 Torres, T. 1990. Étude Paleobotanique du Tertiaire des iles Roi Georges et Seymour, Antarctique. Thèse du Diplóme de Doctorat. Lyon, France, Université Claude-Bernard. Tome 1. $212 \mathrm{pp}$. 
En Argentina y zonas de Chile central (Girarde et al. 1987²), Concepción (Takahashi 1977), isla Quiriquina (Takahashi 1978), Osorno (Troncoso \& Barrera 1980), Navidad (Méon et al. 1994) los registros son mayoritariamente del Terciario.

Según Dettmann (1992), la diversificación de Proteaceae coincide con los cambios en el hábitat asociados con la apertura primaria del océano austral. Además, en áreas de altas latitudes hubo una evolución y diferenciación in situ de grupos australes en la región Australia-Antártica. Se han reconocido dos lugares de evolución y diversificación, que son: el área alrededor del océano austral en formación, el cual se reconoce como centro de diversificación de las Proteaceae; y diametralmente opuesta la región que contiene la parte sur de Sudamérica y la península Antártica, el cual se reconoce también como centro de diversificación de los primeros Nothofagus durante una etapa volcánica y tectónica activa. Según Dettmann (1989) y Askin (1989), la Antártica probablemente sirvió como una ruta de dispersión para varias otros taxa. Los registros en la actualidad indican que varias Proteaceae y Epacridaceae se introdujeron dentro de Australia previo a su último ingreso en el CretácicoTerciario en el oeste de la Antártica.

Hill \& Scriven (1995), indican que la razón de la extinción de un gran número de especies de Proteaceae en el final del Eoceno puede haber sido provocada directa o indirectamente por cambios climáticos, incluyendo un aumento en la abundancia de aves y/o mamíferos polinizadores, un cambio en el tipo de vegetación, que hizo que la polinización anemófila fuera menos competitiva. Bell (1968), considera óptimas las especies para una polinización anemófila, cuando éstas poseen granos de polen entre 20-50 $\mu \mathrm{m}$, mientras que aquellos de mayores dimensiones, están adaptados a polinización ornitófila, mastozoofila o entomófila. En la actualidad, las Proteaceae están adaptadas a una polinización entomófila, ornitófila, quiropterófila, o polinizadas por roedores y marsupiales (Watson \& Dallwitz 1992). Hill (1994), señala la contradicción que existe entre la gran cantidad de granos de polen que se encuentran en los sedimentos fosilíferos y la poca cantidad de macrofósiles encontrados en los

2 Girarde, S., L. Passarelli, C. Guido, M. Castro, M. Tellería \& M. Morbelli 1987. Análisis palinológico de las Proteaceae R. BR. de Argentina. 149-152. In: VII Simposio Argentino de Paleobotánica y Palinología, Buenos Aires, 13-15 Abril 1987, ZLOTOPIORO, Argentina. mismos. Para el autor esta divergencia se explica, por un cambio de estrategia en la polinización. Supone que en el Terciario Inferior la polinización anemófila era significativa en un grupo de Proteaceae, hoy extintas, poseedoras de granos de polen entre 20$40 \mu \mathrm{m}$, mientras todos los demás eran más grandes, incluso mayores a $100 \mu \mathrm{m}$. Efectos indirectos de un cambio climático, indujo a una cambio en la estrategia de polinización (debido a un incremento de polinización zoofila y entomófila), y a un cambio en el tipo de vegetación, que hacía a la polinización anemófila una estrategia menos competitiva. Por ejemplo, si las Proteaceae hubiesen sido árboles sin copa, la polinización anemófila hubiese sido un método adecuado, en bosques abiertos. El desarrollo de una vegetación cerrada significó que un bosque con copa, como los de Nothofagus, Casuarina y coníferas, utilizaran una polinización anemófila, mientras que representantes de taxa que vivían bajo ellos se extinguían.

En general, las Proteaceae actuales están representadas en Chile por seis especies nativas, las cuales tienen la siguiente distribución geográfica, según Donoso (1974)³ y Hoffmann (1982):

- Lomatia dentata (R. et P.) R. Br. "Avellanillo", es una especie endémica de Chile, que crece por ambas cordilleras desde la provincia de Choapa (Región de Coquimbo) hasta la parte norte de la provincia de Chiloé (Región de los Lagos), desde casi el nivel del mar hasta los 1.800 m s.n.m.

- Lomatia hirsuta (Lam.) Diels "Radal", crece en Chile entre Coquimbo y Chiloé, especialmente en los faldeos de ambas cordilleras, entre los 150 y 1.200 m s.n.m. Además, se encuentra en los bosques subantárticos de Argentina, como así también en Perú y Ecuador.

- Lomatia ferruginea (Cav.) R. Br. "Fuinque", es una especie endémica de los bosques subantárticos. En Chile crece desde el sur del río Biobío (Región del Bio Bío) hasta la provincia de Última Esperanza (Región de Magallanes), entre los 5-1.125 m s.n.m. Es particularmente abundante en las provincias de Valdivia a Chiloé. Según Hoffmann (1982), esta especie crece espontáneamente desde la zona costera de Curicó, por el norte, hasta Magallanes, por el sur. En la cordillera de los Andes se le encuentra desde el Biobío.

3 Donoso, C. 1974. Dendrología. Árboles y arbustos chilenos. Manual № 2. Facultad de Ciencias Agrarias y Forestales, Universidad de Chile. $142 \mathrm{pp}$. 
- Gevuina avellana Mol. "Avellano", es un árbol endémico de los bosques subantárticos. En Chile crece ampliamente desde la provincia de Curicó (Región del Maule) hasta las islas Guaytecas (Región de Aisén), especialmente en los faldeos de ambas cordilleras. Según Hoffmann (1982), esta especie tiene como límite norte Valparaíso, por la costa.

- Embothrium coccineum Forst. "Notro", es una especie endémica de los bosques subantárticos. En Chile crece desde la provincia de Curicó hasta la isla Hoste (Región de Magallanes). El área de mayor concentración es la región situada al sur del lago Llanquihue y Chiloé, siendo esta última parte de su centro de dispersión.

- Orites myrtoidea (P. et E.) B. et H. "Radal enano", es una especie altamente endémica y de hábitat estricto, con poblaciones muy endogámicas. Se desarrolla en las laderas rocosas de los cerros, desde el río Maule hasta el Biobío. Variedad chilena. Extremadamente escasa y considerada entre las especies de nuestro país en vías de extinción.

Tomando en consideración el endemismo y la monoespecificidad de las Proteaceae que crecen en Chile, se realizó este estudio que tiene por objetivo la identificación y descripción de granos de polen fósil de Proteaceae, encontrados en rocas sedimentarias de la isla Rey Jorge (Antártica) y de dos localidades de Chile continental. Mediante estudios de morfología comparada se realizó un análisis de las afinidades existentes entre algunos de los ejemplares fósiles y el polen de las especies chilenas.

\section{MATERIAL Y MÉTODOS}

Las muestras de material fósil, se recolectaron en la península Fildes (62² $12^{\prime} S$; $\left.58^{\circ} 56^{\prime} \mathrm{W}\right)$, isla Rey Jorge, archipiélago de las Shetland del Sur Antártica, durante la XXXIII Expedición Científica Antártica 1996/1997, organizada por el Instituto Antártico Chileno. Los sedimentos estudiados provienen de varios puntos de la península Fildes, los que se detallan a continuación (Fig.1): cerro Fósil, localidad fosilífera clásica de la isla Rey Jorge, asignada al PaleocenoEoceno (Torres $1990^{1}$, Torres \& Méon 1990); punta Suffield, asignada al Eoceno-Oligoceno (Torres 1990) ${ }^{1}$, y un afloramiento cercano al refugio ruso, donde no se ha determinado la edad. También se estudiaron sedimentos provenientes de dos localidades terciarias de Chile continental (Fig.1): Matanzas, en la zona central, de la Formación Navidad, asignado al Mioceno Inferior alto hasta el Mioceno Superior inicial, en el acantilado aflorante de la playa de Matanzas; y de los estratos que afloran en el río Las Minas, a 4 km de la ciudad de Punta Arenas, asignado al Eoceno. Las muestras se encuentran depositadas en la Colección Paleobotánica T.Torres del Instituto Antártico Chileno, en el Laboratorio de Paleobotánica en la Facultad de Ciencias Agronómicas, Universidad de Chile.

Para el tratamiento palinológico de los granos de polen fósil se utilizaron los procesos físicos químicos de rutina usados en palinología (Torres $1990^{1}$, Hebel 1997). Basándose en el estudio morfológico con microscopía óptica, se describieron los granos de polen de acuerdo con las siguientes características a evaluar: polaridad, simetría, aperturas (forma, posición, estructura, número), forma, tamaño, estructura y ornamentación de la exina, señaladas por Erdtman (1952), Kapp (1969), Praglowski \& Punt (1973) y Villagrán (1984)4 . El material estudiado está individualizado con las coordenadas entregadas por la lámina universal utilizada en Palinología.

Para determinar la afinidad al polen de Proteaceae actual se utilizó el trabajo realizado por Hebel \& Rojas (2000), el cual describe rigurosa y detalladamente los granos de polen de la familia, tomados de diferentes individuos en diferentes latitudes de acuerdo a la distribución espacial que cada especie posee en Chile.

\section{RESULTADOS}

El barrido de las preparaciones microscópicas de fósiles permitió reconocer, morfológicamente, muchos palinomorfos (tanto esporas como granos de polen) y restos de tejido vascular, pertenecientes a distintas familias, contemporáneas a Proteaceae. La cantidad de especies fósiles afines a Proteaceae encontradas en las distintas áreas de recolección, se elevó a un total de 13, de las cuales ocho pertenecerían a Proteacidites, tres a Propylipollis. Dos pertenecerían a Triorites, cuyo género se encuentra clasificado dentro de otra familia.

De acuerdo con las características evaluadas, la cantidad de ejemplares encontrados y la localidad se lograron las descripciones que se detallan a continuación.

4 Villagran, C. 1984. Curso Electivo de Palinología. Guías de Trabajos Prácticos. Fac. Cs. Básicas y Farmacéuticas, Universidad de Chile, Stgo, Chile. 63 pp. 




Fig. 1. Localidades de recolección de muestras mencionadas en el texto (Fuentes: Cartografía digital ESRI, Antarctic Digital Database v4.1y SIG Fildes INACH/IGM).

(* Acuerdo entre la República de Chile y la República Argentina para precisar el recorrido del límite desde el monte Fitz-Roy hasta el monte Daudet (Buenos Aires, Argentina, 16 de Diciembre de 1998) 


\section{LÁMINA I}
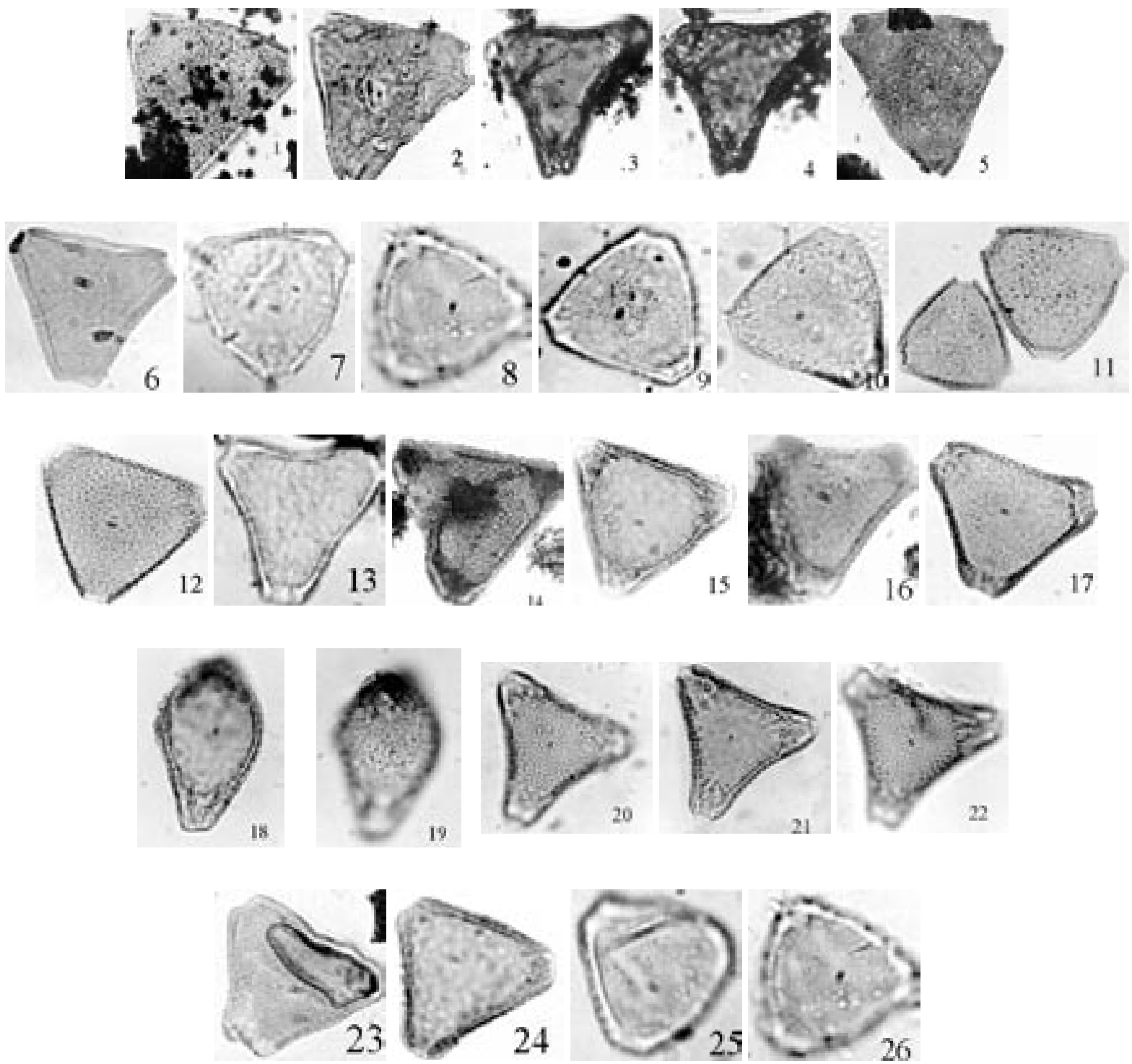

Lámina I. Fig.1 Proteacidites rynthius Stover y Partridge; Fig. 2 Proteacidites sp. 1; Fig.3 y 4 Proteacidites sp. 2; Fig. 5 Proteacidites rectomarginis Cookson; Fig.6 Proteacidites adenanthoides Cookson; Fig.7 Proteacidites cf. subscabratus Couper; Fig.8 Orites myrtoidea (Poepp. \& Endl.) Benth. \& Hooker; Fig.9 y 10 Proteacidites cf. callosus Cookson; Fig. 11 Orites myrtoidea (Poepp. \& Endl.) Benth. \& Hooker; Fig. 12 Lomatia hirsuta

(Lam.) Diels; Fig.13 Cf. Proteacidites pseudomoides Stover 1973; Fig.14 Propylipollis microverrucatus Truswell;

Fig.15 Lomatia ferruginea (Cav.) R.Br. Fig.16 Propylipollis sp. 1; Fig.17 Lomatia hirsuta (Lam.) Diels; Fig.18, 19, 20 y 21 Propylipollis concretus (Harris) Martin y Harris; Fig.22 Gevuina avellana Mol.; Fig.23 Triorites africaensis Jardiné y Magloire; Fig.24 Orites myrtoidea (Poepp. \& Endl.) Benth. \& Hooker; Fig. 25 Triorites harrisii Couper; Fig.26 Orites myrtoidea (Poepp. \& Endl.) Benth. \& Hooker. Todos los ejemplares 1000X. 


\section{DESCRIPCIONES SISTEMÁTICAS}

Según la clasificación de taxa de polen fósil que incluye a las Proteaceae encontradas en los sedimentos en (Martin 1995):

Turma POROSES Naum. Emend. Potonié

Subturma TRIPORINES Naum. Emend. Potonié

a) Proteacidites Cookson emend. Martin \& Harris 1974

b) Propylipollis Martin \& Harris 1974

c) Triorites Cookson ex Couper 1953

a) Proteacidites Cookson emend.

Martin \& Harris 1974

Proteacidites rynthius Stover \& Partridge (Lám. I, Fig. 1)

Descripción. Exomorfología: Isopolar. Simetría radiosimétrica. Aperturas circulares o cercanas a esta forma, posición ecuatorial, estructura simple, triporados, cuyo diámetro puede fluctuar entre 1,4 y 7,7 $\mu \mathrm{m}$. Forma: amb triangular escaleno; lados rectos a débilmente convexos. Estructura de la sexina: semitectada, reticulada, en algunas zonas escabrada. Lúmina variable en tamaño y forma. Lúmina de 0,6 a 4,1 $\mu \mathrm{m}$. Brocato de 0,5 a 3,4 $\mu \mathrm{m}$. Diámetro ecuatorial: 31,5-55,5 $\mu \mathrm{m}$.

Endomorfología: Ectexina y endexina presentes. Es posible identificar con el microcópio óptico la sexina y, más difícil resulta observar la nexina. La nexina posee una densidad promedio de $1 \mu \mathrm{m}$. La sexina a su vez tiene dimensiones en espesor de 0,5 a 1,4 $\mu \mathrm{m}$. La capa interna se adelgaza hacia los poros.

Material estudiado. Preparación microscópica 5, coordenadas: F51/4; M47; M43; S39/2; G39.

Lugar de recolección: punta Suffield, península Fíldes, isla Rey Jorge, Antártica.

Afinidad botánica. Proteaceae

Proteacidites sp. 1

(Lám. I, Fig. 2)

Descripción. Exomorfología: Isopolar. Simetría radiosimétrica. Aperturas de forma circular, posi- ción ecuatorial, estructura simple, triporados, cuyo diámetro puede fluctuar entre 2,4 y 4,1 $\mu \mathrm{m}$. Forma: amb triangular más o menos isósceles, con dos lados y dos alturas semejantes en longitud; lados rectos. Estructura de la sexina: tectada, escabrada con verrucosidades y/o microequinada poco densa. Diámetro ecuatorial: 24,9-30,4 $\mu \mathrm{m}$.

Endomorfología: Ectexina y endexina presentes. Es posible identificar con el microscópio óptico nexina y sexina, con alguna dificultad. La nexina posee una densidad que fluctua entre 0,5 y $1,2 \mu \mathrm{m}$. La sexina a su vez tiene dimensiones en espesor de 0,9 a 1,1 $\mu \mathrm{m}$.

Material estudiado. Preparación microscópica 7, coordenadas: Y42/2.

Lugar de recolección: punta Suffield, península Fíldes, isla Rey Jorge, Antártica.

Afinidad botánica. Proteaceae.

Proteacidites sp. 2

(Lám. I, Figs. 3 y 4)

Descripción. Exomorfología: Isopolar. Simetría radiosimétrica. Aperturas circulares o cercanas a esta forma, posición ecuatorial, estructura simple, triporados, cuyo diámetro puede fluctuar entre 3,5 y $4,1 \mu \mathrm{m}$. Forma: amb triangular; lados rectos a débilmente cóncavos. Estructura de la sexina: semitectada, fina e irregularmente reticulada, en algunas zonas escabrada. Lúmina variable en tamaño y forma. Lúmina de 0,7 a 0,9 $\mu \mathrm{m}$. Brocato de 0,6 a 1,0 $\mu \mathrm{m}$. Diámetro ecuatorial: 37,3-45,1 $\mu \mathrm{m}$.

Endomorfología: Ectexina y endexina presentes. Es posible identificar con el microscópio óptico nexina 1 , nexina 2 y sexina. La nexina 1 posee una densidad de entre 0,3 a 1,7 $\mu \mathrm{m}$. La nexina 2 fluctua entre 0,5 y $0,8 \mu \mathrm{m}$. La sexina, a su vez, tiene dimensiones en espesor de 0,8 a 1,2 $\mu \mathrm{m}$.

Material estudiado. Preparación microscópica 3500(2)TT15, coordenadas: R29/3.

Lugar de recolección: punta Suffield, península Fíldes, isla Rey Jorge, Antártica.

Afinidad botánica. Proteaceae.

Proteacidites rectomarginis Cookson (Lám. I, Fig. 5)

Descripción. Exomorfología: Isopolar. Simetría radiosimétrica. Aperturas circulares a más elongadas, 
posición ecuatorial, estructura simple, triporados, cuyo diámetro puede fluctuar entre 2,3 y 6,8 $\mu \mathrm{m}$. Forma: amb triangular; lados rectos a débilmente convexos. Estructura de la sexina: tectada, escabrada y relativamente uniforme en todo el grano. Diámetro ecuatorial: 37,4-39,4 $\mu \mathrm{m}$.

Endomorfología: Ectexina presentes. Es posible identificar con el microscópio óptico sólo sexina, ésta tiene una dimensión promedio de $1,1 \mu \mathrm{m}$.

Material estudiado. Preparación microscópica 3508 (2), coordenadas: F52/2. Preparación microscópica 3507 (2), coordenadas: J26/2

Lugar de recolección: punta Suffield, península Fíldes, isla Rey Jorge, Antártica.

Afinidad botánica. Proteaceae.

\section{Proteacidites adenanthoides Cookson (Lám. I, Fig. 6)}

Descripción. Exomorfología: Isopolar. Simetría radiosimétrica. Aperturas débilmente elongadas ecuatorialmente, posición ecuatorial, estructura simple, triporados, cuyo diámetro puede fluctuar entre 1,0 y 5,4 $\mu \mathrm{m}$. Forma: amb triangular escaleno; lados rectos a cóncavos. Estructura de la sexina: tectada. Diámetro ecuatorial: 14,7-36,2 $\mu \mathrm{m}$.

Endomorfología: Ectexina y endexina presentes. Es posible identificar con el microcópio óptico la sexina y, más difícil resulta observar la nexina. La nexina posee una densidad que fluctua entre 0,7 y $1,2 \mu \mathrm{m}$. La sexina a su vez tiene dimensiones en espesor de 0,3 a 1,3 $\mu \mathrm{m}$. La capa interna se adelgaza hacia los poros.

Material estudiado. Preparación microscópica 4010, coordenadas: G32/4; M35/3; L53/2.

Lugar de recolección. refugio ruso, península Fíldes, isla Rey Jorge, Antártica.

Afinidad botánica. Proteaceae.

\section{Proteacidites cf. subscabratus Couper (Lám. I, Fig. 7)}

Descripción. Exomorfología: Isopolar. Simetría radiosimétrica. Aperturas circulares, posición ecuatorial, estructura simple, triporados, cuyo diámetro puede fluctuar entre 3,5 y 7,8 $\mu \mathrm{m}$. Forma: amb triangular escaleno a isóceles; dos lados rectos a débilmente convexos. Estructura de la sexina: tectada, escabrada. Diámetro ecuatorial: 29,3-51,9 $\mu \mathrm{m}$.
Endomorfología: Ectexina y endexina presentes. Es posible identificar con el microcópio óptico la sexina y, más difícil resulta observar la nexina. Ésta posee una densidad de 1,2 a 1,8 $\mu \mathrm{m}$. Se produce un engrosamiento de ella hacia los poros. La sexina a su vez tiene dimensiones en espesor de 0,5 a $1,6 \mu \mathrm{m}$.

Material estudiado. Preparación microscópica 3513 (5), coordenadas: D44. Preparación microscópica 3635, coordenadas: O39/1, 1K38/2. Preparación microscópica 4, coordenadas: 3T49/4.

Lugar de recolección: cerro Fósil, península Fíldes, isla Rey Jorge, Antártica. Matanzas, Región de O'Higgins, Chile.

Afinidad botánica. Proteaceae, Orites myrtoidea (Lám. I, Fig. 8).

\section{Proteacidites cf. callosus Cookson (Lám. I, Figs. 9 y 10)}

Descripción. Exomorfología: Isopolar. Simetría radiosimétrica. Aperturas débilmente elongadas ecuatorialmente, posición ecuatorial, estructura simple, triporados, cuyo diámetro puede fluctuar entre 5,8 y 7,0 $\mu \mathrm{m}$. Forma: amb triangular escaleno; lados rectos a convexos. Estructura de la sexina: semitectada, fina e irregularmente reticulada. Lúmina es variable, fluctuando entre 0,7 y 0,9 $\mathrm{mm}$. Brocato entre 0,3 y 0,5 $\mu \mathrm{m}$, de ancho. Diámetro ecuatorial: 39,7-41,6 $\mu \mathrm{m}$.

Endomorfología: Ectexina y endexina presentes. Es posible identificar con el microcópio óptico la nexina y sexina. La nexina posee una densidad que fluctua entre 0,6 y 0,9 $\mu \mathrm{m}$. La sexina a su vez tiene dimensiones en espesor de 0,5 a 0,9 $\mu \mathrm{m}$.

Material estudiado. Preparación microscópica 3635, coordenadas: O43.

Lugar de recolección: Matanzas, Región de O'Higgins, Chile.

Afinidad botánica. Proteaceae, Orites myrtoidea y Lomatia hirsuta (Lám. I, Figs. 11 y 12 , respectivamente). 1973

\section{Cf. Proteacidites pseudomoides Stover}

(Lám. I, Fig. 13)

Descripción. Exomorfología: Isopolar. Simetría radiosimétrica. Aperturas circulares o más elonga- 
das, posición ecuatorial, estructura simple, poros convexos en vista polar, triporados, cuyo diámetro puede fluctuar entre 5,2 y 7,7 $\mu \mathrm{m}$. Forma: amb triangular; lados rectos a cóncavos. Estructura de la sexina: semitectada, irregularmente reticulada. Lúmina variable en tamaño y forma. Lúmina de 0,5 a 2,2 $\mu \mathrm{m}$. Brocato de 0,5 a 0,7 $\mu \mathrm{m}$. Diámetro ecuatorial: $37,5-40,7 \mu \mathrm{m}$.

Endomorfología: Ectexina y endexina presentes. Es posible identificar con el microcópio óptico nexina y sexina. La nexina posee un espesor entre 0,9 a $1,1 \mu \mathrm{m}$. La sexina a su vez tiene dimensiones entre 0,7 a $0,9 \mu \mathrm{m}$.

Material estudiado. Preparación microscópica 3635, coordenadas: N39/3.

Lugar de recolección: Matanzas, Región de O'Higgins, Chile. avellana.

Afinidad botánica. Proteaceae, Gevuina

b) Propylipollis Martin \& Harris, 1974

Propylipollis microverrucatus Truswell

(Lám. I, Fig. 14)

Descripción. Exomorfología: Isopolar. Simetría radiosimétrica. Aperturas débilmente elongadas ecuatorialmente, posición ecuatorial, estructura simple, crassimarginado con solución de la nexina parcial o bien desarrollada, triporados, cuyo diámetro puede fluctuar entre 2,8 y 5,7 $\mu \mathrm{m}$. Forma: amb triangular más o menos isósceles, con dos lados y dos alturas semejantes en longitud; rectos a cóncavos. Estructura de la sexina: tectada, verrucada, en algunas zonas con sexina retículada o con perforaciones. La lúmina, si se presenta, entonces es variable en tamaño y forma. Lúmina entre 0,5 y $2,4 \mu \mathrm{m}$. Brocato entre 0,5 y $1,0 \mu \mathrm{m}$, de ancho. Diámetro ecuatorial: 48,3-51,5 $\mu \mathrm{m}$.

Endomorfología: Ectexina y endexina presentes. Es posible identificar con el microcópio óptico nexina 1 , nexina 2 y sexina, las que se pueden observar difícilmente. La nexina 1 , posee una densidad que fluctúa entre 0,8 y $1,9 \mu \mathrm{m}$. La nexina 2 posee un espesor promedio de $0,5 \mu \mathrm{m}$. La sexina a su vez tiene dimensiones en espesor de 0,7 a $3,2 \mu \mathrm{m}$.

Material estudiado. Preparación microscópica 5, coordenadas: S57/4.
Lugar de recolección: punta Suffield, península Fíldes, isla Rey Jorge, Antártica.

Afinidad botánica. Proteaceae, Lomatia ferruginea (Lám. I, Fig. 15).

\section{Propylipollis sp. 1}

(Lám. I, Fig. 16)

Descripción. Exomorfología: Isopolar. Simetría radiosimétrica. Aperturas de forma circular, posición ecuatorial, estructura simple, crassimarginados circundado por una banda espesa de nexina, bien desarrollada, triporados, cuyo diámetro puede fluctuar entre 1,7 y 7,0 $\mu \mathrm{m}$. Forma: amb triangular escaleno, a veces más o menos isósceles; lados rectos a cóncavos. Estructura de la sexina: tectado, escabrado a finamente reticulado. Diámetro ecuatorial: 34,6-46,6 $\mu \mathrm{m}$.

Endomorfología: Ectexina y endexina presentes. Es posible identificar con el microcópio óptico la nexina y sexina, claramente. La nexina, posee una densidad que fluctua entre 0,5 y 1,0 $\mu \mathrm{m}$. La sexina a su vez tiene dimensiones en espesor de 0,5 a $1,7 \mu \mathrm{m}$.

Material estudiado. Preparación microscópica 4010, coordenadas: G33/2; X56/1.

Lugar de recolección: refugio ruso, península Fíldes, isla Rey Jorge, Antártica.

Afinidad botánica. Proteaceae, Lomatia hirsuta (Lám. I, Fig. 17).

Propylipollis concretus (Harris) Martin \& Harris

(Lám. I, Figs. 18, 19, 20 y 21)

Descripción. Exomorfología: Isopolar. Simetría radiosimétrica. Aperturas de forma circular, posición ecuatorial, estructura simple, crassimarginados, triporados, cuyo diámetro puede fluctuar entre 4,7 y 7,1 $\mu \mathrm{mm}$. Forma: amb triangular más o menos isósceles, con dos lados y dos alturas semejantes en longitud; rectos a cóncavos, oblado. Estructura de la sexina: semitectado, reticulado, heterobrocado. Lúmina variable en tamaño y forma. Lúmina entre 0,9 y $2,5 \mu \mathrm{mm}$. Brocato entre 0,5 y $1,0 \mu \mathrm{m}$, de ancho. Diámetro ecuatorial: 43,2-60,3 $\mu \mathrm{m}$. Diámetro polar: $33,2 \mu \mathrm{m}$.

Endomorfología: Ectexina y endexina presentes. Es posible identificar claramente con el 
microcópio óptico la nexina 1 , nexina 2 y sexina. La nexina 1 , posee una densidad que fluctua entre 0,7 y $1,5 \mu \mathrm{m}$. La nexina 2, varía entre 0,6 a 1,5 $\mu \mathrm{m}$. La sexina a su vez tiene dimensiones en espesor de 0,7 a $1,4 \mu \mathrm{m}$.

Material estudiado. Preparación microscópica 2 (1), coordenadas: U29/4, V30/2, U30/2, R35/4, S36.

Lugar de recolección: Matanzas, Matanzas, Región de O'Higgins, Chile.

Afinidad botánica. Proteaceae, Gevuina avellana (Lám. I, Fig. 22).

\section{c) Triorites Cookson ex Couper 1953}

Triorites africaensis Jardiné \& Magloire (Lám. I, Fig. 23)

Descripción. Exomorfología: Isopolar. Simetría radiosimétrica. Aperturas más elongadas o subcirculares, posición ecuatorial, estructura simple, triporados, cuyo diámetro puede fluctuar entre 2,0 y 4,5 $\mathrm{mm}$. Forma: amb triangular; lados rectos a cóncavos. Estructura de la sexina: semitectada, fina e irregularmente reticulada. Lúmina variable en tamaño y forma. Lúmina de 0,5 a 0,6 $\mu \mathrm{m}$. Brocato de 0,2 a 0,6 $\mu \mathrm{m}$. Diámetro ecuatorial: 28,2-35,4 $\mu \mathrm{m}$.

Endomorfología: Ectexina y endexina presentes. Es posible identificar con el microcópio óptico nexina y sexina. La nexina posee una densidad entre 1,0 a $1,1 \mu \mathrm{m}$. La sexina a su vez tiene dimensiones en espesor de 0,4 a 1,3um.

Material estudiado. Preparación microscópica 4010, coordenadas: N32/2.

Lugar de recolección: refugio ruso, península Fildes, isla Rey Jorge, Antártica.

Afinidad botánica. Proteaceae, Orites myrtoidea (Lám. I, Fig. 24).

\section{Triorites harrisii Couper}

(Lám. I, Fig. 25)

Descripción. Exomorfología: Isopolar. Simetría radiosimétrica. Aperturas subcirculares, posición ecuatorial, estructura simple, triporados, cuyo diámetro puede fluctuar entre 2,7 y 2,9 $\mu \mathrm{m}$. Forma: amb triangular; lados rectos a convexos. Estructura de la sexina: tectada, escabrada, con pequeñas verrucosidades. Diámetro ecuatorial: 18,8-19,9 $\mu \mathrm{m}$.
Endomorfología: Ectexina presentes. Es posible identificar con microcópio óptico solamente la sexina, la cual tiene un espesor promedio de 0,5 $\mu \mathrm{m}$.

Material estudiado. Preparación microscópica 3635, coordenadas: L37/1. Preparación microscópica 4038, coordenadas: T46.

Lugar de recolección: Matanzas, Región de O'Higgins, Chile. Punta Arenas, Región de Magallanes, Chile.

Afinidad botánica. Proteaceae, Orites myrtoidea (Lám. I, Fig. 26).

En los cuadros 1, 2 y 3, se resumen las características de las especies fósiles afines a Proteaceae, descritas anteriormente, agrupadas de acuerdo a las localidades de recolección.

Según la identificación y descripción de los granos de polen fósil afines a las Proteaceae, se elaboró la tabla 4.

\section{DISCUSIÓN Y CONCLUSIONES}

En general, los granos de polen de Propylipollis, corresponden a aquellos granos que poseen un post atrium y/o se encuentran frecuentemente anillados. Por lo cual, Dudgeon (1983), prefirió el término "crassimarginados" indicando una marcada densidad de la nexina alrededor del poro, donde la zona de post atrium presenta una parcial o total disolución o corrosión de la nexina. Truswell \& Owen (1988), indican que Propylipollis fue establecido para separar granos de polen triporados con supuesta afinidad a Proteaceae, en donde la exina está modificada cerca de los poros, de aquellos granos en que la exina permanece sin modificaciones. Por otro lado, Proteacidites se caracteriza por sus aperturas sin la presencia de una zona post atrium.

Específicamente para Propylipollis concretus, hallado en Matanzas, los granos de polen encontrados tienen dimensiones mayores a los referidos por Milne (1988), y tienen mayor semejanza con aquellos a los cuales los denomina como especímenes "raramente grandes". Difiere, además, en la estructura de la sexina que describe como escabrada, lo que sería un error en la observación o decripción, ya que en las ilustraciones se muestra una estructura semitectada y reticulada, más que escabrada. Además, se encuentra alguna afinidad con la especie denominada Propylipollis reticuloscabratus (Harris) Martin \& Harris, citada por Milne (1988). 
TABLA 1. Resumen de las características de las especies fósiles encontradas en los sedimentos del Mioceno de Matanzas.

\begin{tabular}{|c|c|c|c|c|c|c|}
\hline Carac/sp. & $\begin{array}{l}\text { Propylipollis } \\
\text { concretus }\end{array}$ & $\begin{array}{l}\text { Proteacidites } \\
\text { cf. callosus }\end{array}$ & $\begin{array}{c}\text { Proteacidites cf. } \\
\text { subscabratus }^{1}\end{array}$ & $\begin{array}{c}\text { Cf. Proteacidites } \\
\text { pseudomoides }\end{array}$ & $\begin{array}{l}\text { Proteacidites } \\
\text { rectomarginis }\end{array}$ & Triorites harrisii ${ }^{2}$ \\
\hline Polaridad & Isopolar & Isopolar & Isopolar & Isopolar & Isopolar & Isopolar \\
\hline Simetría & Radial & Radial & Radial & Radial & Radial & Radial \\
\hline Apertura & $\begin{array}{l}\text { Triporado } \\
4,7-7,1 \mu \mathrm{m}\end{array}$ & $\begin{array}{l}\text { Triporado } \\
5,8-7,0 \mu \mathrm{m}\end{array}$ & $\begin{array}{l}\text { Triporado } \\
3,5-7,8 \mu \mathrm{m}\end{array}$ & $\begin{array}{l}\text { Triporado } \\
5,2-7,7 \mu \mathrm{m}\end{array}$ & $\begin{array}{l}\text { Triporado } \\
2,3-6,8 \mu \mathrm{m}\end{array}$ & $\begin{array}{l}\text { Triporado } \\
2,7-2,9 \mu \mathrm{m}\end{array}$ \\
\hline Forma & $\begin{array}{l}\text { Triangular más o } \\
\text { menos isósceles }\end{array}$ & $\begin{array}{l}\text { Triangular } \\
\text { escaleno }\end{array}$ & $\begin{array}{l}\text { Triangular escaleno } \\
\text { a isósceles }\end{array}$ & Triangular & Triangular & Triangular \\
\hline $\mathrm{P}$ & $33,2 \mu \mathrm{m}$ & - & - & - & - & - \\
\hline $\mathrm{E}$ & $51,0 \mu \mathrm{m}$ & $40,4 \mu \mathrm{m}$ & $40,4 \mu \mathrm{m}$ & $26,4 \mu \mathrm{m}$ & $42,2 \mu \mathrm{m}$ & $19,5 \mu \mathrm{m}$ \\
\hline $\mathrm{P} / \mathrm{E}$ & Oblado & - & - & - & - & - \\
\hline $\begin{array}{l}\text { Estruct. } \\
\text { sexina }\end{array}$ & Reticulado & $\begin{array}{l}\text { Irregularmente } \\
\text { reticulado }\end{array}$ & $\begin{array}{l}\text { Irregularmente } \\
\text { reticulado }\end{array}$ & Tectada & $\begin{array}{l}\text { Reticulada y en } \\
\text { zonas escabrada }\end{array}$ & $\begin{array}{l}\text { Escabrada, pequeñas } \\
\text { verrucosidades }\end{array}$ \\
\hline Lúmina & $0,9-2,5 \mu \mathrm{m}$ & $0,7-0,9 \mu \mathrm{m}$ & $0,7-0,9 \mu \mathrm{m}$ & - & $0,6-4,1 \mu \mathrm{m}$ & - \\
\hline Brocato & $0,5-1,0 \mu \mathrm{m}$ & $0,3-0,5 \mu \mathrm{m}$ & $0,3-0,5 \mu \mathrm{m}$ & - & $0,5-3,4 \mu \mathrm{m}$ & - \\
\hline
\end{tabular}

Carac/sp.: Características por columnas y especies abreviadas en estudio por filas. Estruct. sexina: Estructura de la sexina. P: Diámetro polar. E: Promedio diámetro ecuatorial. P/E: Relación diámetro polar: diámetro ecuatorial.

${ }^{1}$ También en Cerro Fósil. ${ }^{2}$ También en Punta Arenas.

TABLA 2. Resumen de las características de las especies fósiles encontradas en sedimentos de punta Suffield, península Fíldes, isla Rey Jorge.

\begin{tabular}{|c|c|c|c|c|c|}
\hline Carac/sp. & $\begin{array}{l}\text { Propylipollis } \\
\text { microverrucatus }\end{array}$ & Proteacidites rynthius & $\begin{array}{l}\text { Proteacidites } \\
\text { sp. } 1\end{array}$ & $\begin{array}{l}\text { Proteacidites } \\
\text { sp. } 2\end{array}$ & $\begin{array}{l}\text { Proteacidites } \\
\text { rectomarginis }\end{array}$ \\
\hline Polaridad & Isopolar & Isopolar & Isopolar & Isopolar & Isopolar \\
\hline Simetría & Radial & Radial & Radial & Radial & Radial \\
\hline Apertura & $\begin{array}{l}\text { Triporado } \\
2,8-5,7 \mu \mathrm{m}\end{array}$ & $\begin{array}{l}\text { Triporado } \\
1,4-7,7 \mu \mathrm{m}\end{array}$ & $\begin{array}{l}\text { Triporado } \\
2,4-4,1 \mu \mathrm{m}\end{array}$ & $\begin{array}{l}\text { Triporado } \\
3,5-4,1 \mu \mathrm{m}\end{array}$ & $\begin{array}{l}\text { Triporado } \\
2,3-6,8 \mu \mathrm{m}\end{array}$ \\
\hline Forma & $\begin{array}{l}\text { Triangular más o } \\
\text { menos isóceles }\end{array}$ & Triangular escaleno & $\begin{array}{l}\text { Triangular más o } \\
\text { menos isóceles }\end{array}$ & Triangular escaleno & Triangular \\
\hline E & $49,5 \mu \mathrm{m}$ & $42,2 \mu \mathrm{m}$ & $28,4 \mu \mathrm{m}$ & $41,2 \mu \mathrm{m}$ & $42,2 \mu \mathrm{m}$ \\
\hline Ornam. & verrucosidades & s/o & $\begin{array}{l}\text { verrucosa o } \\
\text { microequinada } \\
\text { poco densas }\end{array}$ & s/o & s/o \\
\hline $\begin{array}{l}\text { Estruct. } \\
\text { sexina }\end{array}$ & $\begin{array}{l}\text { Tectada con algunas } \\
\text { zonas perforadas }\end{array}$ & $\begin{array}{l}\text { Reticulada y en } \\
\text { zonas escabrada }\end{array}$ & Tectada, escabrada & $\begin{array}{l}\text { Fina e irregularmente } \\
\text { reticulado, escabrado } \\
\text { en algunas zonas }\end{array}$ & $\begin{array}{l}\text { Reticulada y en } \\
\text { zonas escabrada }\end{array}$ \\
\hline Lúmina & $0,5-2,4 \mu \mathrm{m}$ & $0,6-4,1 \mu \mathrm{m}$ & & $0,7-0,9 \mu \mathrm{m}$ & $0,6-4,1 \mu \mathrm{m}$ \\
\hline Brocato & $0,5-1,0 \mu \mathrm{m}$ & $0,5-3,4 \mu \mathrm{m}$ & & $0,6-1,0 \mu \mathrm{m}$ & $0,5-3,4 \mu \mathrm{m}$ \\
\hline
\end{tabular}

Carac/sp.: Características por columnas y especies abreviadas en estudio por filas. Ornam.: Ornamentación. Microequin.: Microequinada. Estruct. sexina: Estructura de la sexina. s/o: sin ornamentación E: Promedio diámetro ecuatorial. 
TABLA 3. Resumen de las características de las especies fósiles encontradas en sedimentos del refugio ruso, península Fildes, isla Rey Jorge.

\begin{tabular}{llll}
\hline \multicolumn{1}{c}{ Carac/sp } & \multicolumn{1}{c}{ Propylipollis sp. 1} & Proteacidites adenanthoides & Proteacidites africaensis \\
\hline Polaridad & Isopolar & Isopolar & Isopolar \\
Simetría & Radial & Radial & Radial \\
& Triporado & Triporado & Triporado \\
Apertura & $1,7-7,0 \mu \mathrm{m}$ & $1,0-5,4 \mu \mathrm{m}$ & $2,0-4,5 \mu \mathrm{m}$ \\
Forma & Triangular escaleno & Triangular escaleno & Triangular \\
E & $39,3 \mu \mathrm{m}$ & $26,4 \mu \mathrm{m}$ & $39,3 \mu \mathrm{m}$ \\
Estruct. sexina & Tectada, escabrada & Tectada & Escabrada \\
\hline
\end{tabular}

Carac/sp.: Características por columnas y especies abreviadas en estudio por filas. E: Promedio diámetro ecuatorial. Estruct. sexina: Estructura de la sexina.

TABLA 4. Resumen de las especies encontradas según lugar de obtención, edad de la localidad fosilífera y afinidad a las especies actuales de Proteaceae.

\begin{tabular}{|c|c|c|c|}
\hline Lista de especies encontradas & Lugar & Edad & Afinidad con especies actuales \\
\hline Propylipollis concretus & Matanzas & Mioceno & Gevuina avellana \\
\hline Proteacidites cf. callosus & Matanzas & Mioceno & $\begin{array}{l}\text { Lomatia hirsuta } \\
\text { Orites myrtoidea }\end{array}$ \\
\hline Cf. Proteacidites pseudomoides & Matanzas & Mioceno & Gevuina avellana \\
\hline Proteacidites cf. subscabratus & $\begin{array}{l}\text { Matanzas } \\
\text { Cerro Fósil }\end{array}$ & $\begin{array}{c}\text { Mioceno } \\
\text { Paleoceno-Eoceno }\end{array}$ & Orites myrtoidea \\
\hline Proteacidites harrisii & $\begin{array}{c}\text { Matanzas } \\
\text { Punta Arenas }\end{array}$ & $\begin{array}{c}\text { Mioceno } \\
\text { Eoceno }\end{array}$ & Orites myrtoidea \\
\hline Propylipollis microverrucatus & Punta Suffield & Eoceno-Oligoceno & Lomatia ferruginea \\
\hline Proteacidites rynthius & Punta Suffield & Eoceno-Oligoceno & $\mathrm{S} / \mathrm{a}$ \\
\hline Proteacidites sp.1 & Punta Suffield & Eoceno-Oligoceno & $\mathrm{S} / \mathrm{a}$ \\
\hline Proteacidites sp. 2 & Punta Suffield & Eoceno-Oligoceno & $\mathrm{S} / \mathrm{a}$ \\
\hline Proteacidites rectomarginis & Punta Suffield & Eoceno-Oligoceno & $\mathrm{S} / \mathrm{a}$ \\
\hline Propylipollis sp.1 & Refugio Ruso & Cretácico Superior-Terciario? & Lomatia hirsuta \\
\hline Proteacidites adenanthoides & Refugio Ruso & Cretácico Superior-Terciario? & $\mathrm{S} / \mathrm{a}$ \\
\hline Proteacidites africaensis & Refugio Ruso & Cretácico Superior-Terciario? & $\mathrm{S} / \mathrm{a}$ \\
\hline
\end{tabular}

Por su semejanza con ejemplares encontrados por Troncoso \& Barrera (1980), en Osorno, asignados al Eoceno, se determinó a uno de los ejemplares como Proteacidites cf. callosus. Sin embargo, esos ejemplares poseen una exina con rangos mínimo y máximo menores. El diámetro ecuatorial también es de menor tamaño.
El ejemplar determinado como cf. Proteacidites pseudomoides es semejante a aquellos publicados por Stover \& Partridge (1982) y Mohr (1990). Sin embargo, el ejemplar estudiado posee lados más cóncavos y poros más elongados. También, se encuentra una afinidad con la especie determinada como Proteacidites parvus Cookson, publicada por 
Harris (1965), pero la especie estudiada difiere de ella, por presentar un retículo más grueso.

El ejemplar de este estudio afín a Proteacidites cf. subscabratus presenta aberturas simples con un pequeño engrosamiento de la nexina en la zona cercana a los poros y su forma es triangular lo que lo hace afín a las Proteaceae y no a familias como Onagraceae o Myricaceae, las cuales presentan aberturas en forma de aro alrededor del poro, con lo que sufren un pequeño engrosamiento y elongación de éste. Este ejemplar es semejante a lo publicado por Baldoni \& Barreda (1986) y difiere de lo descrito por Milne (1988), Troncoso \& Barrera (1980) y Dolding (1992).

El ejemplar determinado como Propylipollis microverrucatus, se debe a sus aberturas crassimarginadas y su sexina verrucada. Difiere del determinado por Truswell \& Owen (1988), por la presencia de retículo en ciertas áreas y no en la totalidad del grano. Sin embargo, estos autores admiten la presencia de estructuras con pequeñas perforaciones. El ejemplar estudiado tiene dimensiones mayores que el publicado por ellos.

Proteacidites rynthius, posee aperturas sin la presencia de una zona post atrium. El ejemplar es triangular, con una estructura de la sexina desde reticulada a escabrada, lo que lo hace semejante a los publicados por Stover \& Partridge (1982), Milne (1988) y Mohr (1990), siendo más parecida a las publicadas por este último.

Proteacidites sp.1, parece semejante a $P$. rectomarginis Cookson, determinada, además, por Torres (1990) $)^{1}$, pero de mucho menor tamaño y con sexina tectada.

Proteacidites sp.2, parece semejante a las publicadas por Takahashi (1978), Troncoso \& Barrera (1980), Mildenhall \& Suggate (1981). El tamaño de los ejemplares es mayor a los descritos por estos autores, por lo que no se le otorgó una afinidad a P. minor, Takahashi (1978) y/o P. minimus Couper, Mildenhall \& Suggate (1981). Además, el tamaño de la exina es notable, el desarrollo del retículo es menor. Por estas razones, se supone más afín a $P$. concretus.

Proteacidites rectomarginis Cookson, ya había sido ilustrada por Torres (1990) ${ }^{1}$, por lo que ahora, se proporciona la descripción de ésta, para posteriores trabajos. La especie parece muy semejante a la publicada por Baldoni (1987), que la clasifica como $P$. rectus Pocknall \& Mildenhall, sin embargo, la especie en estudio es de mayor tamaño.

Los ejemplares clasificados como a Propylipollis sp. son muy parecidos a aquellos encontrados por Harris (1965), que lo identifica como Proteacidites scaboratus. Milne (1988), lo clasifica como Propylipollis sp. B, por tener aperturas crassimarginadas. Sin embargo, sólo un ejemplar de los publicados por Milne, es semejante a los obtenidos en este estudio.

Los ejemplares determinados como Proteacidites adenanthoides, parecen semejantes a lo publicado por Cookson (1953) y, a lo identificado por Archangelsky (1973), como Proteacidites sp. El último autor describe la sexina con columelas pequeñas formando un microretículo, lo cual no fue posible observar en los ejemplares estudiados. Difiere completamente (en tamaño, estructura de la sexina, lados más rectos, etc.), a la publicada por Harris (1965), que se cree está determinada o publicada en forma errónea, debido a que el autor se basa en trabajos realizados por Cookson. Se debe hacer notar que la que se publica como afín a $P$. adenanthoides, tiene una mayor afinidad a P. cf. crassus Cookson, publicada con este nombre por Cookson (1953) y, la que es más afín a la especie descrita, la denomina como $P$ cf. P. rectomarginus Cookson. Por el trabajo de Stover \& Partridge (1973), los ejemplares estudiados pudieran pertenecer sólo al tipo $P$. adenanthoides, ya que sus ejemplares poseen una lúmina y brocato, fácilmente observable al microscopio, y con un retículo fino cercano a los poros.

Cookson et al (1954), citado por Harris (1965), determina a Triorites como afín a Myricaceae, probablemente por poseer sus ejemplares poros más complejos. El ejemplar publicado por Harris, presenta el mismo tamaño que nuestro el ejemplar estudiado afin a Triorites, sin embargo, difiere en el espesor de la sexina, la estructura de las aberturas y la ornamentación (pequeñas verrucosidades). Según Troncoso \& Barrera (1980), la especie Triorites harrisii fue nuevamente clasificada como Haloragacidites harrisii (Couper) Harris. Dada la gran variabilidad presente en los géneros actuales de Proteaceae, específicamente lo observado en Orites (Hebel \& Rojas 2000), se determinaron como afines a Proteacidites los que previamente habían sido determinados como Triorites. En este trabajo se propone que varias especies descritas anteriormente como pertenecientes al género Triorites, pueden tener afinidad con las Proteaceae. 
Triorites africaensis, se asemeja bastante a uno descrito por Jardiné \& Magloire (1965), los cuales indican una probable afinidad con Proteaceas primitivas.

\section{Afinidades a especies actuales}

Basados en el trabajo descriptivo de los granos de polen de las especies actuales de Proteaceae provenientes de diversas localidades de Chile obtenidos de distintos años de recolección de Hebel \& Rojas (2000), se infiere que siete de las trece especies fósiles presentadas, tienen un alto grado de semejanza a especies de Proteaceae chilenas actuales. Así se establece una clara afinidad de:

- Propylipollis microverrucatus con Lomatia ferruginea;

- Propylipollis concretus y Proteacidites pseudomoides con Gevuina avellana;

- Proteacidites aff callosus y Propylipollis sp.1 con Lomatia hirsuta;

- Proteacidites aff callosus, Proteacidites cf. subscabratus y Proteacidites harrisii con Orites myrtoidea.

La causa de que más de una especie fósil sea afín a una especie actual se debe a la alta variabilidad en cuanto a tamaño, ornamentación, etc. que presentan los granos de polen actuales considerando año de recolección de las muestras y localidades distribuidas latitudinalmente.

Para el resto de los palinomorfos encontrados no se pudo reconocer una afinidad específica para un género dentro de las Proteaceae actuales, ya que son similares a cinco de las seis especies presentes en Chile, por la forma triangular, los lados rectos a cóncavos, sus poros circulares a elongados, la densidad de la sexina, las dimensiones de diámetro ecuatorial. Sin embargo, no concuerdan en la ornamentación y la estructura de la sexina. Específicamente, algunos de los ejemplares fósiles son tectados, mientras que los actuales son semitectados $y$, si son tectados entonces tienen ornamentación. Estos ejemplares fósiles podrían representar una especie "tipo", antigua, por la estructura de la sexina, la cual corresponde a un carácter primitivo, de donde se desarrollaron todos los demás tipos de estructura de la sexina.

El establecimiento de una vinculación entre el polen fósil Terciario y las actuales Proteaceae que crecen en el país, se logró, principalmente, en muestras de sedimentos provenientes del Mioceno de Chile continental y del Eoceno Superior de la isla Rey Jorge, Antártica. Esto indicaría, que sólo los granos de polen encontrados en sedimentos más modernos son asimilables a las especies hoy existentes en el país. Las formas halladas en sedimentos más antiguos, serían menos parecidas a las especies actuales por: (a) degradación de la pared a través de los años lo cual no permite hacer comparaciones, (b) diferencia de los granos de polen debido a que las especies fósiles están extintas y no tienen relación con las existentes hoy, (c) las especies fósiles encontradas en estos sedimentos no están representadas en la flora actual chilena, sino que tienen mayor relación con las especies actuales de puntos geográficos como Australia y Nueva Zelandia, específicamente demostrado con especies de Beaupreaidites. Se infiere, por lo tanto, que al menos a partir del Mioceno, existían ancestros de tres de los cuatro géneros que crecen en Chile. De lo anterior, se deduce que algunas variables pueden ser más estables en el tiempo, como el tamaño, la forma, las aperturas, etc. y menos estables como una degradación de la densidad o espesor de las paredes, ornamentación de los granos de polen, etc. Así, de este análisis, se puede inferir la importancia de establecer una metodología que permita evaluar la norma de degradación de los granos de polen en el tiempo. Esto evitaría la proliferación de especies fósiles determinadas sobre la base de palinomorfos.

\section{AGRADECIMIENTOS}

Al Instituto Antártico Chileno, por su apoyo en la realización de este trabajo al permitirnos participar en la Expedición Científica Antártica XXXIII.

A la Dr. Henriette Méon por sus constructivas críticas y el apoyo prestado en la elaboración de algunas preparaciones microscópicas.

A la Sección Botánica del Museo Nacional de Historia Natural de Santiago, en especial a Gloria Rojas, Mélica Muñoz, Inés Meza y Elisabeth Barrera.

Al Sr. Ricardo Jaña investigador INACH/ CEQUA por la elaboración de los mapas para esta publicación.

A todos los revisores, por sus comentarios y sugerencias. 


\section{LITERATURA CITADA}

Archangelsky, S.1973. Palinología del Paleoceno de Chubut. I. Descripciones Sistemáticas. Ameghiniana. 4(10): 339-399.

Askin, R. 1989. Endemism and heterochroneity in the Late Cretaceous (Campanian) to Paleocene palynofloras of Seymour Island, Antarctica: implications for origins, dispersal and paleoclimates of southern floras. Geological Society Special Publication. 47:107-119.

Askin, R. 1990. Campanian to Paleocene spore and pollen assemblages of Seymour Island, Antarctica. Review of Palaeobotany and Palynology. 65:105-113.

Baldoni, A. \& V. Barreda 1986. Estudio palinológico de las formaciones López de Bertodano y Sobral, isla Vicecomodoro Marambio, Antártida. Boletín Instituto de Geociencia, Universidad de Sao Paulo. 17: 89-98.

Baldoni, A. 1987. Estudios palinológicos de la zona de Colloncura, Provincia de Neuquén, sobre elementos del Terciario Inferior y redepositados del Cretácico Inferior. Revista Española de Micropaleontología. 19(3): 367-411.

Carpenter, R.J. 1994. Cuticular morphology and aspects of the ecology and fossil history of north Queensland rainforest Proteaceae. Botanical Journal of Linnean Society. 116: 249-303.

Cookson, I. 1953. Difference in microspore composition of some samples from a bore at Comaum, South Australia. Australian Journal of Botany. 1(3): 462-473.

Dettmann, M. E. 1989. Antarctica: Cretaceous cradle of austral temperate rainforests? Geological Society Special Publication. 47: 89-105.

Dettmann, M. E. 1992. Structure and floristics of Cretaceous vegetation of southern Gondwana: implications for angiosperm biogeography. Palaeobotanist 41: 224-233.

Dolding, P.J.D. 1992. Palynology of the Marambio Group (Upper Cretaceous) of northern Humps Island. Antarctic Science. 4(3): 311-326.

Dudgeon, M.J. 1983. Eocene pollen of probable proteaceus affinity from the Yaamba Basin, Central Queensland. In: Dorothy Hill Jubilee Memoir, J. Roberts and P.A.Jell Eds. Memoirs of the Association of Australasian Palaentologists 1: 339-362.

Duigan, S. 1950. A Catalogue of the Australian Tertiary Flora. Proceeding of the Royal Society Victoria. 62: 1-56.

Erdtman, G. 1952. Pollen Morphology and Plant Taxonomy. Angiosperms. Ed. Almqvist \& Wiksells, Stockholm. The Chronica Botanica CO.: Waltham, Mass. 539 pp.

Harris, W. 1965. Basal Tertiary microfloras from the Princetown area, Victoria, Australia. Palaeontographica Abt. B. 115: 75-106.

Hebel, I. \& G. Rojas 2000. Morfología de los granos de polen de especies de la familia Proteaceae presentes en Chile. Bol. Museo Nac. de Hist. Nat. 49: 51-72.

Hill, R.S. \& J.L. Scriven 1995. The angiospermdominated woody vegetation of Antarctica: a review. Review of Palaeobotany and Palynology. 86:175-198.

Hoffmann, A. 1982. Flora Silvestre de Chile. Zona araucana. 3 ${ }^{\text {a }}$. Fundación Claudio Gay. Santiago. Chile. 258 pp.

Jardiné, S. \& L. Magloire 1965. Palynologie et stratigraphie du Crétacé des Bassins du Sénégal et de côte d'ivoire. Mem. Bur. Rech. Geol. Miniere 32:188-245.

Jordan, G.J., M.K. Macphail, R. Barnes \& R.S. Hill 1995. An Early to Middle Pleistocene Flora of Subalpine Affinities in Lowland Western Tasmania. Australian Journal of Botany. 43: 231-242.

Kapp, R. 1969. How to Know Pollen and Spores. WM.C. Brown, Dubuque, Iowa, USA. 249 pp.

Li, H. 1992. Early Tertiary Palaeoclimate of King George Island, Antarctica-Evidence from the Fossil Hill Flora. 371-375. In: Recent Progress in Antarctica Earth Science. Yoshida, Tokio, Japan.

Martin, A.R.H. 1995. Palaeogene proteaceous pollen and phylogeny. Alcheringa 19: 27-40.

Méon, H., T. Torres \& R. Martínez-Pardo 1994. Sporopollinic analysis in the Navidad Formation near Navidad (Chile). In: Actas $7^{\circ}$ Congreso Geológico Chileno, Concepción. Vol.1: 488-490.

Mildenhall, D.C. \& R.P. Suggate 1981. Palynology and age of the Tadmor Group (Late Mioce- 
ne-Pliocene) and Porika Formation (early Pleistocene), South Island, New Zealand. New Zealand Journal of Geology and Geophysics. 24: 515-528.

Milne, L. A. 1988. Palynology of a late Eocene lignitic sequence from the western margin of the Eucla Basin, Western Australia. Memoir of the Association of Australasian Palaentologists. 5: 285-310.

Mohr, B. 1990. Eocene and Oligocene Sporomorphs and Dinoflagellate Cysts from Leg 113 Drill Sites, Weddell Sea, Antarctica. Proceedings of the Ocean Drilling Program, Scientific Results. 113: 595-612.

Pole, M.S. 1992. Eocene vegetation from Hasties, noth-eastern Tasmania. Australian Systematic Botany. 5: 431-475.

Praglowski, J. \& W. Punt 1973. An elucidation of the microreticulate structure of the exine. Grana. 13: 45-50.

Stover, L. \& A. Partridge 1973. Tertiary and Late Cretaceous spores and pollen from the Gippsland Basin, Southeastern Australia. Royal Society of Victoria Proceedings. 85(2): 237-286.
Stover, L. \& A. Partridge 1982. Eocene spore-pollen from the Werillup formation, Western Australia. Palynology. 6: 69-95.

Stuchlik, L. 1981. Tertiary pollen spectra from the Ezcurra Inlet Group of Almiralty Bay, King George Island, South Shetland Islands, Antarctica. Studia Geologica Polonica. 72: 109-132.

Takahashi, K. 1977. Palynology of the lower Tertiary Concepción Formation, Central Chile. Trans. Proc. Palaeont. Soc. Japan n.s., 106: 71-87.

Takahashi, K. 1978. Upper Cretaceous palynofossils from Quiriquina Island, Chile. Journal of Palynology. 14(1): 30-49.

Torres, T. \& H. Méon 1990. Estudio palinológico preliminar de cerro Fósil, península Fildes, isla Rey Jorge, Antártica. Ser. Cient. INACH. 40: 21-39.

Troncoso, A. \& E. Barrera 1980. Polen del Eoceno de Osorno. Boletín Museo Nacional de Historia Natural, Chile. 37:179-203.

Truswell, E. M. \& J. A. Owen 1988. Eocene pollen from Bungonia, New South Wales. Memoir of the Association of Australasian Palaentologists. 5: 259-284. 